\title{
Influence of Parameter Variation on Electromechanical Admittance Spectrum of a Multiple Degree of Freedom System
}

\author{
Dai Zishu \\ Department of Geotechnical Engineering \\ Tongji University \\ Shanghai, China \\ daizish@hotmail.com \\ Cai Yongchang \\ Department of Geotechnical Engineering \\ Tongji University \\ Shanghai, China \\ yccai@tongji.edu.cn
}

\author{
Han Yiying \\ Department of Geotechnical Engineering \\ Tongji University \\ Shanghai, China \\ Yiyin.Han@hotmail.com \\ Tian Jiao \\ Department of Geotechnical Engineering \\ Tongji University \\ Shanghai, China \\ Tianjiao@163.com
}

\begin{abstract}
Electromechanical impedance technique is a very important research field in the structural health monitoring. This paper intends to study the mechanism of some interesting phenomena in the application of this technique, such as variation rules in electrical conductance spectra at high frequency range are different from those at low frequency range. This work will help to understand the test results of electromechanical impedance measurement better. A PZT patch attached to a multiple degree of freedom system model is established and its electrical admittance expression is also derived. By adjusting the parameters of one changing component in the multiple degree of freedom system, the rules how mass, stiffness and damping parameters influence the electrical conductance spectrum are investigated. The calculation results shows that those conductance peaks formed by PZT patch modals change in different patterns with the variation of component parameters if they are on the different side of resonant frequency of changing component. In addition, the equation of resonant frequency of PZT patch and the changing component will result in the corresponding conductance peak being divided into two.
\end{abstract}

Keywords- PZT; MDOF; EMI; conductance; spectrum;

\section{INTRODUCTION}

During last two decades, electromechanical impedance (EMI) technique has become an important branch of structural health monitoring (SHM) and non-destructive test (NDE) [1]. In this technique, piezoceramic (PZT) patches are employed as active sensor-actuators attached to or embedded in the host mechanical structure. The dynamic interaction between the PZT patches and the host mechanical structure will result in a unique electrical admittance spectrum, revealing the physical and mechanical property variation in the host structure. Sun et al. [2] introduced this technique into damage detection of civil engineering components such as pipeline system, truss structure and steel bridge sections. Taylor et al. [3] monitored fatigue failure of a wind turbine rotor blade under cyclic loads based on EMI technique. Soh and Bhalla [4] built an empirical relationship between admittance resonant frequency and concrete strength.

Although EMI technique has been proved to be effective in monitoring mechanical state of aerospace and civil engineering members, there are still some interesting phenomena of admittance spectra in the application of EMI technique which have not been explained theoretically well. For example, in the research of assessment dental implant stability [5], it was observed that the peaks at low frequency denoted an opposite trend to those at high frequency. And sometimes it was also found that after great change in the host structure, there were some pairs of 'twin peaks', while the other peaks stay unchanged.

In this paper, an analytical expression of electrical admittance of a PZT patch attached to a multiple degree of freedom (MDOF) system will be derived. The way that parameters of the mechanical components in the MDOF system influence electrical admittance of the PZT patch will be analyzed and discussed, which can be used to explain those mentioned interesting phenomena in the application of EMI technique.

\section{PZT-STRUCTURE INTERACTION MODEL}

The piezoceramic patch is the key component of EMI technique. Piezoceramic is a kind of noncentosymmetric crystal with piezoelectric effect, which means the material generates an electric field inside when applied a mechanical stress and conversely undergo mechanical deformation when subjected to electric field. The direct and converse effect piezoelectric effect of a thin PZT patch with polarization in the thickness can be described by [6] 


$$
\begin{aligned}
& \mathrm{D}_{3}=\overline{\varepsilon_{33}^{\mathrm{T}}} \mathrm{E}_{3}+\mathrm{d}_{31} \mathrm{~T}_{1} . \\
& \mathrm{S}_{1}=\mathrm{T}_{1} / \overline{\mathrm{Y}^{\mathrm{E}}}+\mathrm{d}_{31} \mathrm{E}_{3} .
\end{aligned}
$$

where subscript ' 3 ' represents the axis along the thickness of the PZT patch and subscript ' 1 ' or ' 2 ' represent the axes in the plane of the patch. D3 is electric displacement vector over the surface of the PZT patch, $\mathrm{E}_{3}$ is applied external electric field vector; $S_{1}$ and $T_{1}$ is the strain and stress vector in the plane of the PZT patch. $\overline{\varepsilon_{33}^{T}}=\varepsilon_{33}^{\mathrm{T}}(1-\delta \mathrm{j})$ is complex dielectric permittivity at constant stress; $d_{31}$ is the piezoelectric strain coefficients and $\overline{Y^{E}}=Y^{E}(1+\eta j)$ is the complex Young's modulus. $\delta$ and $\eta$ denote the dielectric loss factor and the mechanical loss factor.

Providing that the PZT patch is a one-dimensional rod undergoing axial vibrations driven by high frequency (30 $\mathrm{kHz}-400 \mathrm{kHz}$ ) alternating current, the dynamic governing equation is described as

$$
\overline{Y^{E}} \frac{\partial^{2} u}{\partial x^{2}}=\rho \frac{\partial^{2} u}{\partial t^{2}}
$$

where $\mathrm{u}$ is axial displacement of any point in the patch and $\rho$ is PZT's density. The force-displacement relationship of endpoint of PZT patch can be written as

$$
\overline{\mathrm{Y}^{\mathrm{E}}} \frac{\partial^{2} \mathrm{u}}{\partial \mathrm{x}^{2}}=\rho \frac{\partial^{2} \mathrm{u}}{\partial \mathrm{t}^{2}}
$$

where $\mathrm{u}$ is axial displacement of any point in the patch and $\rho$ is PZT's density. The force-displacement relationship of endpoint of PZT patch can be written as

$$
\mathrm{F}_{(\mathrm{x}=\mathrm{l})}=-\mathrm{Z} \dot{\mathrm{u}}_{(\mathrm{x}=\mathrm{l})} .
$$

where $\mathrm{Z}$ is the drive point mechanical impedance of the mechanical system. The actuator impedance $Z_{a}$ is defined as

$$
\mathrm{Z}_{\mathrm{a}}=\frac{\mathrm{F}_{(\mathrm{x}=\mathrm{l})}}{\dot{\mathrm{u}}_{(\mathrm{x}=\mathrm{l})}}=\frac{\kappa w \mathrm{~h} \overline{\mathrm{Y}^{\mathrm{E}}}}{\mathrm{j} \omega \tan (\kappa \mathrm{l})} .
$$

where $\kappa=\omega \sqrt{\rho / \overline{Y^{E}}}$ is wave number. According to the relationship between electric current and electric displacement,

$$
I=j \omega \int D_{3} d A
$$

the electric admittance expression (reciprocal of electrical impedance) can be derived as [7]

$$
\mathrm{Y}(\omega)=\omega \mathrm{jl} \frac{\mathrm{wl}}{\mathrm{h}}\left[\overline{\varepsilon_{33}^{\mathrm{T}}}-\mathrm{d}_{31}^{2} \overline{\mathrm{Y}^{\mathrm{E}}}+\left(\frac{\mathrm{Z}_{\mathrm{a}}}{\mathrm{Z}+\mathrm{Z}_{\mathrm{a}}}\right) \mathrm{d}_{31}^{2} \overline{\mathrm{Y}^{\mathrm{E}}}\left(\frac{\tan \kappa \mathrm{l}}{\kappa \mathrm{l}}\right)\right]
$$

where $1, \mathrm{w}, \mathrm{h}$ are the length, width and thickness of the PZT patch as shown in Fig.1.

\section{MECHANICAL IMPEDANCE OF MDOF}

As seen in Fig .1, the MDOF system studied in this paper consists of two mechanical components connected in series, both of which have a mass element, a stiffness element and a damping element.

Component 1 is attached to the PZT patch, whose electromechanical characteristic has been described in the previous chapter. The other endpoint of Component 2 is fixed. In this study, the parameters of Component 1 are unchanged, while the parameters of Component 2 are allowed to vary arbitrarily. This model represents a wide range of applications in EMI technique, such as variation of steel member's boundary conditions [8] and an embedded aluminum rod to monitor concrete strength gain [9].

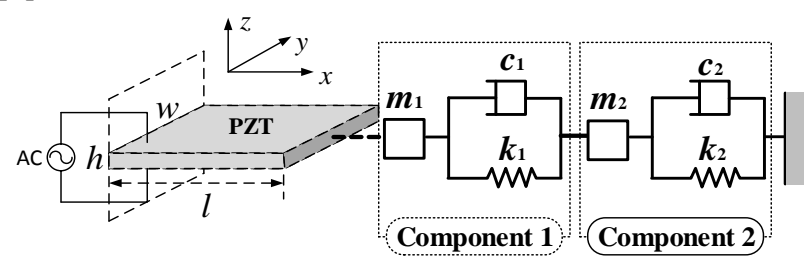

Figure 1. PZT attached to a MDOF system.

The mechanical impedance of Component 1 and Component 2 can be derived as

$$
\begin{aligned}
& \mathrm{z}_{1}=\mathrm{c}_{1}+\mathrm{j} \omega \mathrm{m}_{1}+\mathrm{k}_{1} / \mathrm{j} \omega . \\
& \mathrm{z}_{2}=\mathrm{c}_{2}+\mathrm{j} \omega \mathrm{m}_{2}+\mathrm{k}_{2} / \mathrm{j} \omega .
\end{aligned}
$$

where $Z_{1}$ is the mechanical impedance of Component 1 and $Z_{2}$ is the mechanical impedance of Component 2; $\mathrm{m} 1$, $\mathrm{k} 1, \mathrm{c} 1$ are the mass, stiffness and damping parameters of Component 1 respectively, and $\mathrm{m} 2, \mathrm{k} 2$, c 2 are the mass, stiffness and damping parameters of Component 2 respectively. $\omega$ is the circular frequency of the external cyclical force.

According to the theory of mechanical impedance, the overall mechanical impedance of components in series can be expressed as follows.

$$
\mathrm{Z}_{\mathrm{s}}=\mathrm{Z}_{1} \mathrm{Z}_{2} /\left(\mathrm{Z}_{1}+\mathrm{Z}_{2}\right)
$$

Substituting (10) into (8), the electrical admittance of the MDOF system shown in Fig .1 can be derived as

$$
\mathrm{Y}(\omega)=\omega \mathrm{j} \frac{\mathrm{wl}}{\mathrm{h}}\left[\overline{\varepsilon_{33}^{\mathrm{T}}}-\mathrm{d}_{31}^{2} \overline{\mathrm{Y}^{\mathrm{E}}}+\frac{\mathrm{Z}_{\mathrm{a}}}{\mathrm{Z}_{\mathrm{s}}+\mathrm{Z}_{\mathrm{a}}} \mathrm{d}_{31}^{2} \overline{\mathrm{Y}^{\mathrm{E}}}\left(\frac{\operatorname{tan\kappa l}}{\kappa \mathrm{l}}\right)\right] .
$$

\section{EFFECT OF PARAMETERS VARIATION ON THE CONDUCTANCE SPECTRA}

In most cases, the conductance (real part of electrical admittance) is more sensitive than the susceptance (imaginary part of electrical admittance) to the state variation of host structure, because the mechanical impedance variation resulted from change in the host structure is mainly reflected in the real part of admittance [10]. Therefore, in this paper only electrical conductance spectra are investigated.

A reasonable set of physical parameters of a PZT patch is listed in TABLE I. which will be used in the following analytical study. 
TABLE I. $\quad$ PARAMETERS OF PZT PATCH

\begin{tabular}{|c|c|c|c|c|}
\hline l (m) & w (m) & h (m) & $d_{31}(m / V)$ & $\overline{\mathrm{Y}^{\mathrm{E}}}\left(\mathrm{N} / \mathrm{m}^{2}\right)$ \\
\hline 0.05 & 0.02 & 0.002 & $-166 \mathrm{E}-12$ & $6.3 \mathrm{E} 10$ \\
\hline \multicolumn{2}{|c|}{$\rho\left(\mathrm{kg} / \mathrm{m}^{3}\right)$} & $\varepsilon_{33}(\mathrm{~F} / \mathrm{m})$ & $\delta$ & $\eta$ \\
\hline \multicolumn{2}{|c|}{7650} & $1.5 \mathrm{E}-8$ & 0.012 & 0.001 \\
\hline
\end{tabular}

A set of original parameters of Component 1 and Component 2 is listed in TABLE II., which make the resonant frequency of Component 1 and Component 2 be at $100 \mathrm{kHz}$ and $60 \mathrm{kHz}$, and the damping ratio of Component 1 and Component 2 be 0.01 and 0.05 respectively.

TABLE II. ORGINAL PARAMETERS OF COMPONENT 1 AND 2

\begin{tabular}{|c|c|c|c|c|c|}
\hline \multicolumn{3}{|c|}{ Component 1 } & \multicolumn{3}{c|}{ Component 2 } \\
\hline $\mathbf{m}_{\mathbf{1}}$ & $\mathbf{k}_{\mathbf{1}}$ & $\mathbf{c}_{\mathbf{1}}$ & $\mathbf{m}_{\mathbf{2}}$ & $\mathbf{k}_{\mathbf{2}}$ & $\mathbf{c}_{\mathbf{2}}$ \\
\hline $1 \mathrm{E}-3$ & $3.94 \mathrm{E} 8$ & 12.57 & $1 \mathrm{E}-2$ & $1.42 \mathrm{E} 9$ & 376.99 \\
\hline
\end{tabular}

It should be noted that, the parameters of Component 1 are constant, while the parameters of Component 2 listed above are for reference only. When the components are with the parameters in TABLE II, the MDOF system is in its 'original state'.

Fig .2 shows the electrical conductance in the original state. It is found that at $100 \mathrm{kHz}$ (resonant frequency of Component 1) there exists a peak. However, there exist few more peaks on either sides of it. When compared with mechanical impedance of PZT, it is obvious that those peaks are formed by PZT modals.

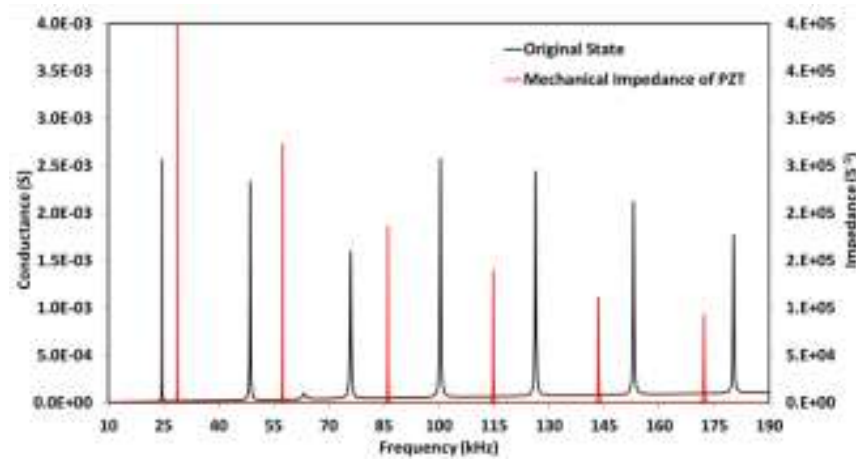

Figure 2. Conductance in original state and impedance of PZT.

\section{A. Conductance Spectrum in Free State}

In this section, the conductance spectrum of PZT attached to the MDOF system in original state will be compared with that in the 'free state'. The 'free state' means parameters of Component 2 are close to zero.

Fig .3 compares the electrical conductance spectra in original state and free state. The peak values in the free state are much higher that those in original state. In addition, the peaks in free state have larger frequency interval.

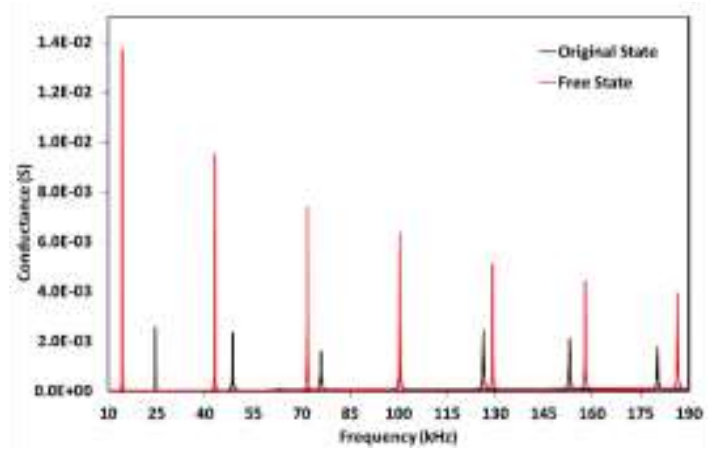

Figure 3. Conductance in original state and free state.

\section{B. Resonant Frequency of Component 2 conincide with that of PZT modal}

Let the parameters of Component 2 be those listed in TABLE III., which make its resonant frequency be at 43 $\mathrm{kHz}$ coincident with that of one order PZT modal. This state is named as 'coincident state'.

TABLE III. PARAMETERS OF COMPONENT 2 IN COINCIDENT STATE

\begin{tabular}{|c|c|c|}
\hline \multicolumn{3}{|c|}{ Component $\mathbf{2}$} \\
\hline $\mathbf{m}_{\mathbf{2}}$ & $\mathbf{k}_{\mathbf{2}}$ & $\mathbf{c}_{\mathbf{2}}$ \\
\hline $1 \mathrm{E}-2$ & $6.41 \mathrm{E} 8$ & 253.2 \\
\hline
\end{tabular}

The electrical conductance in this state is calculated from (11), and compared with original state in Fig .4. It is found that the conductance peak at $43 \mathrm{kHz}$ is divided into two peaks and peak values of both are much smaller than the original one. Thus, the cause of 'twin peaks' in the applications of EMI on crack detection can be explained as the damage lowers the resonant frequency coincident with that of PZT patch.

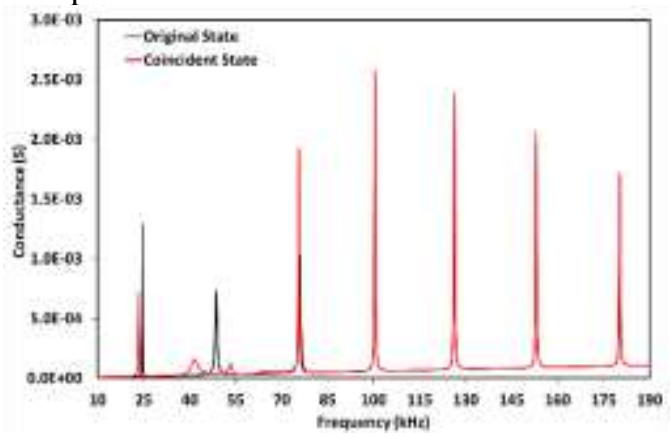

Figure 4. Conductance in original state and coincident state.

\section{Effect of Mass Parameter Variation}

In this section, the mass parameter in Component 2 will fluctuate by $30 \%$ and the effect of mass parameter variation on conductance spectrum is investigated.

It is observed in Fig .5(a) that the conductance peaks on the left of Component 2's resonant frequency $(60 \mathrm{kHz})$ show different variation pattern to that on the right. As seen in Fig .5(b)-(c), with the increase of mass, the peak at $49 \mathrm{kHz}$ moves leftwards with peak value descending, while the peak at $76 \mathrm{kHz}$ moves leftwards with peak value ascending. However, from Fig .5(d), the conductance peak formed by the modal of Component 1 at $100 \mathrm{kHz}$ stays nearly unchanged. 

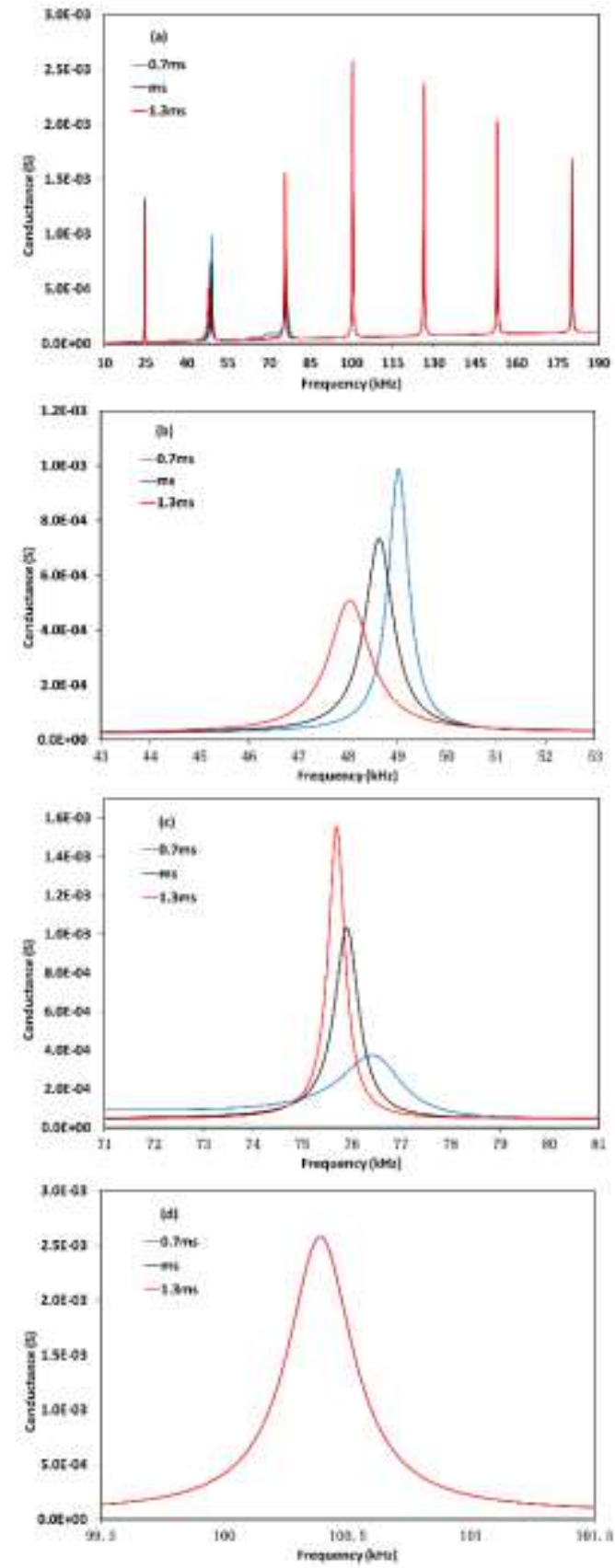

Figure 5. Effect of mass variation on conductance. (a) Overall variation (b) Enlarged view of peaks at $49 \mathrm{kHz}$. (c) Enlarge view of peaks at 76 $\mathrm{kHz}$. (d) Enlarge view of peaks at $100 \mathrm{kHz}$.

\section{Effect of Stiffness Parameter Variation}

In this section, the stiffness parameter in Component 2 will fluctuate by $30 \%$ and the effect of stiffness parameter variation on conductance spectrum is investigated.

It is observed in Fig .6(a) that the conductance peaks on the left of Component 2's resonant frequency $(60 \mathrm{kHz})$ show different variation pattern to that on the right. As seen in Fig .6(b)-(c), with the increase of stiffness, the peak at $49 \mathrm{kHz}$ moves rightwards with peak value ascending, while the peak at $76 \mathrm{kHz}$ moves rightwards with peak value descending. However, from Fig .6(d), the conductance peak formed by the modal of Component 1 at $100 \mathrm{kHz}$ stays nearly unchanged.
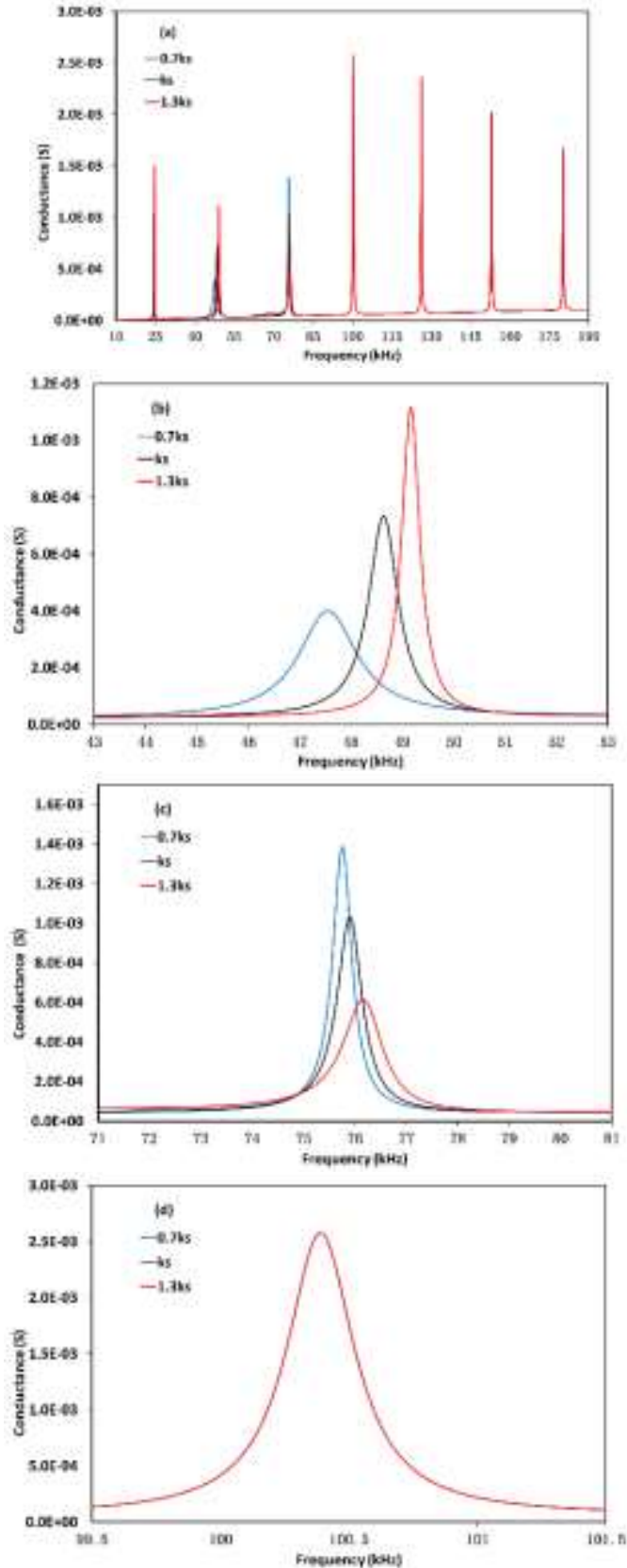

Figure 6. Effect of stiffness variation on conductance. (a) Overall variation. (b) Enlarged view of peaks at $49 \mathrm{kHz}$. (c) Enlarge view of peaks at $76 \mathrm{kHz}$. (d) Enlarge view of peaks at $100 \mathrm{kHz}$.

\section{E. Effect of Damping Parameter Variation}

In this section, the damping parameter in Component 2 will fluctuate by $30 \%$ and the effect of damping parameter variation on conductance spectrum is investigated.

As seen in Fig .7(a)-(d) that, except the peak formed by Component 1 modal, all the other peak values decrease with the increase of damping. The peak formed by Component 1 is unchanged. 

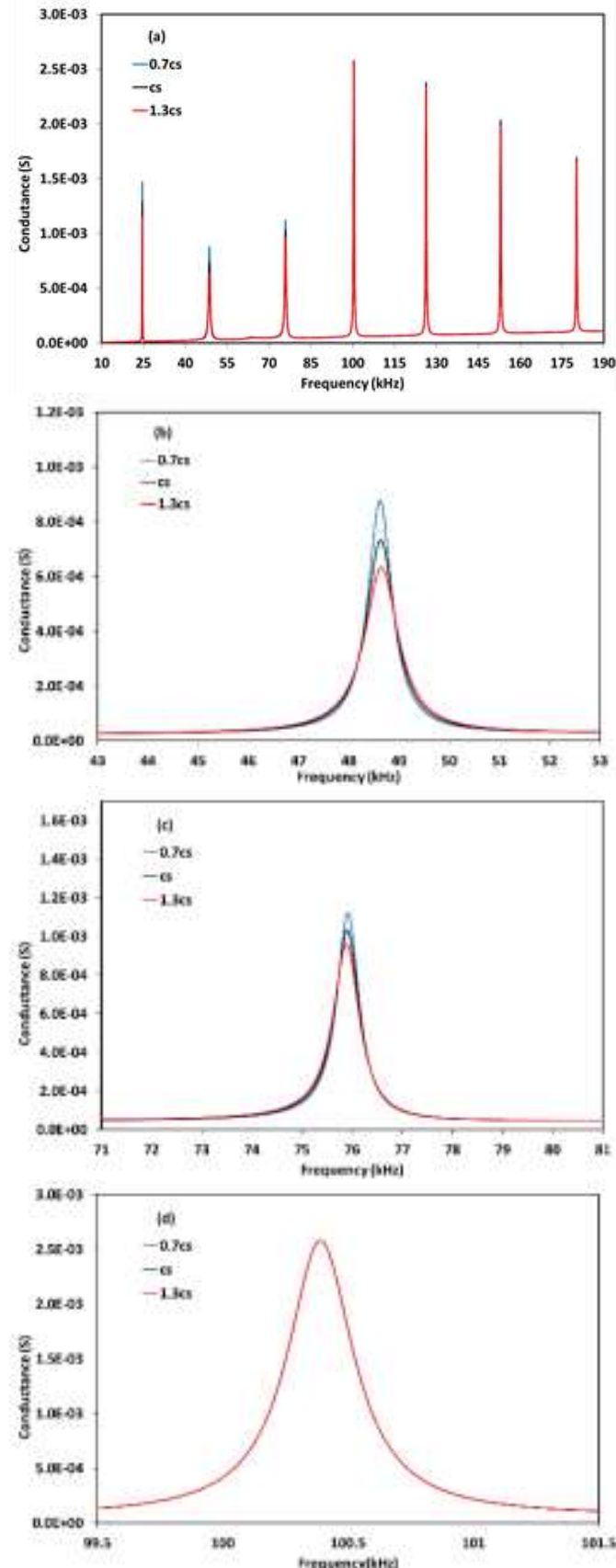

Figure 7. Effect of mass variation on conductance. (a) Overall variation. (b) Enlarged view of peaks at $49 \mathrm{kHz}$. (c) Enlarge view of peaks at 76 $\mathrm{kHz}$. (d) Enlarge view of peaks at $100 \mathrm{kHz}$.

\section{CONCLUSION}

This paper has investigated the cause of some interesting phenomena of electrical conductance spectrum in the application of EMI technique by analyzing a PZT patch attached to a multiple degree of freedom system. By adjusting parameters of one changing component in the MDOF system and calculating corresponding electrical conductance spectra, several variation rules has been obtained and summarized as following:

- There are two kinds of peaks in the conductance spectrum. One kind is formed by the modals of components in the MDOF system and the other kind is formed by the modals of PZT patch.
- When parameters of one component are close to zero, the peak value of conductance will become much larger than before.

- When resonant frequency of one component is coincident with that of one modal of PZT patch, the conductance peak formed by this modal will be divided into two peaks and value of both peaks will decrease.

- The conductance peaks formed by modals of one component are not sensitive to the parameter variation of another component. All the peaks formed by PZT patch modals can be broken into two categories by the resonant frequency of the changing component. When mass parameters increase, those peaks with lower frequency will move leftwards with peak value going down, while those peaks with higher frequency will move leftwards with peak value going up. When the stiffness parameters increase, the peaks will move rightwards with peak value going up, while those peaks with higher frequency will move rightwards with peak value going down. When the damping parameters increase, the peaks will not move in the frequency, but the peak value will decrease.

\section{ACKNOWLEDGMENT}

This research was financially supported by the National Natural Science of China, NSFC(11472194), National Basic Research Program of China (973 Program: 2011CB013800), and New Century Excellent Talents Project in China (NCET-12-0415).

\section{REFERENCES}

[1] C. K. Soh, Y. Yang, and S. Bhalla, Smart Materials in Structural Health Monitoring, Control and Biomechanics, 1st ed., Hangzhou: Zhejiang University Press, 2012, pp.18-19

[2] F. P. Sun, Z. A. Chaudhry, C. A. Rogers, M. Majmundar, and C. Liang, "Automated real-time structure health monitoring via signature pattern recognition.", Proc. SPIE 2443, Smart Structures and Materials 1995: Smart Structures and Integrated Systems, SPIE, May. 1995, pp. 236-247, doi:10.1117/12.208261.

[3] S. G. Taylor, G. Park, K. M. Farinholt, and M. D. Todd "Diagnostics for piezoelectric transducers under cyclic loads deployed for structural health monitoring applications," Smart Materials and Structures, vol. 22(2), Feb. 2013, pp. 025024, doi:10.1088/0964-1726/22/2/025024.

[4] C. K. Soh, and S. Bhalla, "Calibration of piezo-impedance transducers for strength prediction and damage assessment of concrete," Smart Materials and Structures, vol. 14(4), August 2005, pp. 671, doi:10.1088/0964-1726/14/4/026.

[5] G. Boemio, P. Rizzo, L. Nardo, " Assessment of dental implant stability by means of the electromechanical impedance method," Smart Materials and Structures, vol. 20(4), April 2011, pp. 045008, doi:10.1088/0964-1726/20/4/045008

[6] T. Ikeda, Fundamentals of piezoelectricity, Oxford: Oxford university press, 1996, pp. 120-122.

[7] C. Liang, F. P. Sun, and C. A. Rogers. "Coupled electromechanical analysis of adaptive material systems - determination of the actuator power consumption and system energy transfer," Journal of Intelligent Material Systems and Structures, vol. 5(1), 1994, pp. 12-20.

[8] Z. Luo, H. Cao, L. Meng, and L. Lin, "Influences of mechanical contact on damage evaluation with electromechanical impedance technique," Journal of Intelligent Material Systems and Structures, July 2013, pp. 1-11, doi: 10.1177/1045389X13493356.

[9] R. Tawie, and H. K. Lee, "Monitoring the strength development in concrete by EMI sensing technique," Construction and Building 
Materials, vol. 24(9), Sept. 2010, pp: 1746-1753.

[10] S. Bhalla, A. Naidu, and C. K. Soh, "Influence of structureactuator interactions and temperature on piezoelectric mechatronic signatures for NDE." Proc. SPIE 5062, Smart Materials, Structures, and Systems, SPIE, Oct. 2003, pp. 263-269, doi: $10.1117 / 12.514762$ 